\title{
Apical Hypertrophic Cardiomyopathy Presenting as Recurrent Unexplained Syncope
}

\author{
Yusuf Kasirye, MD; Janaki Ram Manne, MD; Narendranath Epperla, MD; \\ Sowjanya Bapani, MD; and Romel Garcia-Montilla, MD
}

\begin{abstract}
Apical hypertrophic cardiomyopathy $(\mathrm{AHC})$ is a rare variant of hypertrophic cardiomyopathy. Since its description by Sakamoto in 1976 in Japanese patients, our understanding of this entity has evolved. Although cardiac magnetic resonance imaging has emerged as the gold standard for diagnosing $\mathrm{AHC}$, clinical attention must be drawn to the unique electrocardiographic features that provide the initial clues to making the diagnosis. In this case, we present a 47 -year-old man with $\mathrm{AHC}$ who presented with recurrent syncope, but anomalies on his electrocardiogram went unnoticed on two clinical encounters. He was subsequently admitted to our service and rapidly diagnosed after we observed the very classical findings in the plain twelve lead electrocardiogram done at the time of admission. In a clinical encounter involving a patient presenting with recurrent syncope, special attention must be focused on the electrocardiogram to decipher the unique diagnostic features it might show.
\end{abstract}

Keywords: Apical hypertrophic cardiomyopathy; Syncope; Electrocardiogram; Diagnosis

Corresponding Author:

Yusuf Kasirye, MD

Department of Internal Medicine

Marshfield Clinic

50 Sherry Avenue

Park Falls, WI 54552 USA

Tel: 7|5-762-729|

Fax: 715-389-5757

Email: kasirye.yusuf@marshfieldclinic.org

Received: December 14, 2010

Revised: April 27, 20II

Accepted: May II, 201I

doi: $10.3121 / \mathrm{cmr} .2011 .986$ 47-year-old man was referred from an outside facility with complaints of recurrent falls. He had been referred to a neurologist for evaluation of recurrent unexplained syncope. Each event was non-convulsive, random, and preceded by a sudden loss of balance that would progress to falling due to altered mental status. There was no apparent triggering factor, post-event confusion, or loss of either bowel or urinary continence. He had been evaluated twice at a local healthcare facility for these falls, but no cause was identified.

He was referred to us on the third presentation after suffering multiple facial and thoracic injuries. He had no evident cardiopulmonary symptoms. Because he was adopted, he had no knowledge of any family history history of cardiac events. His past medical history included bipolar disorder, tobacco abuse, alcohol dependence, and atypical facial pain. His home medications were carbamazepine, lamotrigine, olanzepine, diazepam, and venlafaxine. The only recent change in his medications was a carbamazepine dose reduction because a provider at an outside facility had thought that this might be contributing to his symptoms. On review of his past medical records (more than 10 years prior), left ventricular hypertrophy had been mentioned, but it had not been followed-up in subsequent evaluations. Previous computerized tomography (CT) of the head and neck had been unremarkable.

Clinically, the patient was alert, awake, and oriented. Blood pressure was 123/63 $\mathrm{mmHg}$, pulse 83 beats/minute, temperature $99.6^{\circ} \mathrm{F}$, respirations 20 breaths/ minute, and oxygen saturation $95 \%$ on room air. He had no orthostatic hypotension. The physical examination was normal except for the multiple facial swellings and left-sided chest wall tenderness he had sustained from the falls. 


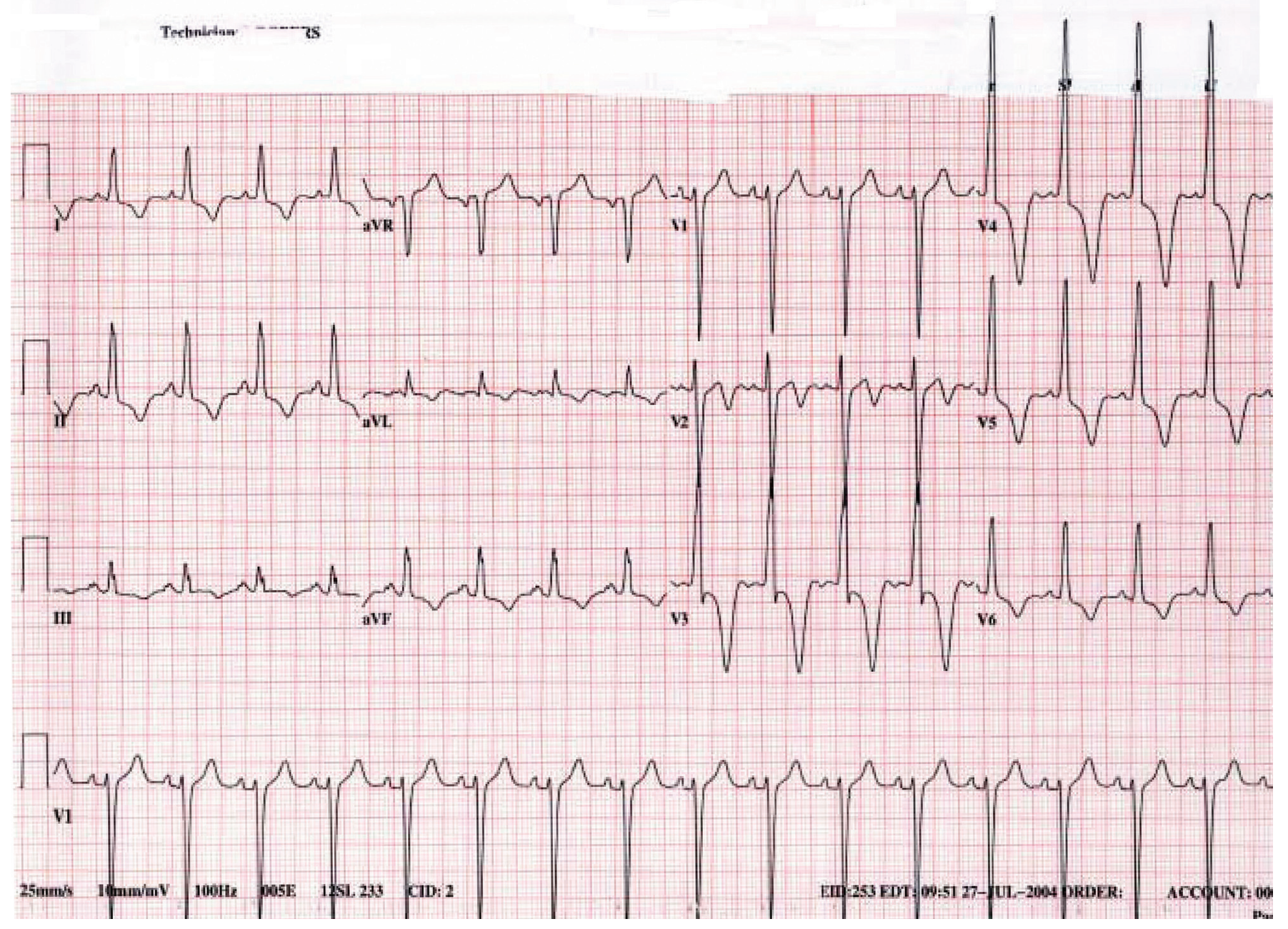

Figure 1. Resting ECG showing left ventricular hypertrophy, high QRS voltage in precordial leads (RV5+SV1=48 mm), QRS interval (80 msec), giant T-wave inversions in V3-V4 (>10 mm), and diffuse absence of septal Q-waves in I, II, III, aVF, aVL, and V3 to V6. ST-segment depression $(\geq 1 \mathrm{~mm}$ ) can also be observed in precordial leads V3 to V6. These features are descriptive of apical hypertrophic cardiomyopathy.

Resting electrocardiogram (ECG) showed left ventricular hypertrophy, high QRS voltage in precordial leads (RV5+SV1=48 mm; QRS interval $80 \mathrm{msec}$ ), giant T-wave inversions in V3-V4 (>10 mm), and diffuse absence of septal Q-waves in I, II, III, aVF, aVL, and V3 to V6. An ST segment depression (equal or greater than $1 \mathrm{~mm}$ ) can also be observed in precordial leads V3 to V6 (figure 1). These features satisfy the diagnostic criteria of left ventricular hypertrophy per both Sokolow-Lyon voltage and Romhilt-Estes point score criterion and are specifically consistent with the pattern of apical hypertrophic cardiomyopathy (AHC)., ${ }^{1,2}$

Significant laboratory results included hemoglobin $8.9 \mathrm{~g} / \mathrm{dL}$ $(14.2 \mathrm{~g} / \mathrm{dL}$ six months earlier), with the remainder of the complete blood count, basic metabolic panel, toxicology screen, urinalysis, and cardiac enzymes within normal range. Chest radiograph and CT scan showed multiple left-sided rib fractures with a large left-sided hemothorax. A neurologist evaluated the patient and concluded that this was not seizurelike activity.
In the absence of a demonstrable neurological cause for his symptoms, and based on the quite striking findings on the ECG, a cardiogenic cause was suspected. The physical injuries were attributed to trauma. A conventional twodimensional trans-thoracic echocardiography was initially done showing hypertrophic cardiomyopathy involving the caudal two-thirds of the left ventricle with no valvulopathy (figure 2A). However, a subsequent, more detailed contrastenhanced echocardiography revealed apical hypertrophy with a left ventricular aneurysm (figure 2B). Based on the clinical presentation, the striking ECG manifestations (mainly in the precordial leads), and the presence of an apical aneurism, a left-sided cardiac catheterization was done, demonstrating a $90 \%$ stenosis in the posterior descending artery with no angiographic evidence of coronary artery disease in the left coronary system. The ventriculogram depicted a classic "ace of spades" appearance of the left ventricle consistent with AHC. Cardiac magnetic resonance imaging (MRI) confirmed $\mathrm{AHC}$ as seen with an apical aneurysm (figure $2 \mathrm{C}$ ). Based on this evidence, the clinical conclusion was that this patient was having recurrent syncopal episodes due to AHC. 

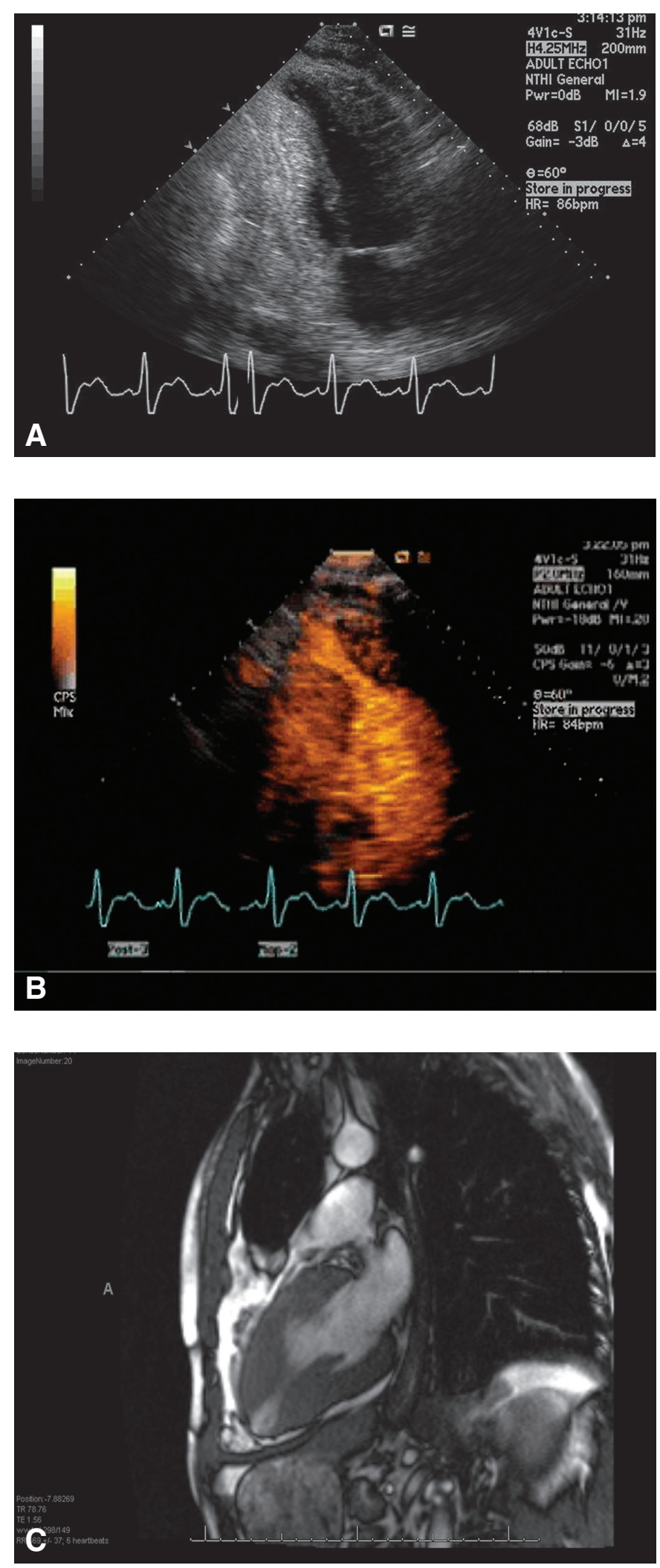

Figure 2. (A) Transthoracic echocardiography apical window images showing apical hypertrophy in diastole. (B) Contrastenhanced transthoracic echocardiography apical window revealing hypertrophy with left ventricular apical aneurysm seen in early diastole. (C) Apical hypertrophy cardiomyopathy with an apical aneurysm as seen on cardiac MRI during diastole.
In view of the recurrent syncope and confirmed diagnosis of $\mathrm{AHC}$, the patient had placement of a dual-chamber cardioverter defibrillator for prophylaxis against sudden cardiac death. Percutaneous coronary intervention to correct the significant right coronary stenosis was not considered due to absence of clinically evident angina and the presence of a large hemothorax that eventually required chest tube placement. The significant drop in hemoglobin was attributed to this hemothorax. The rest of his traumatic injuries were managed conservatively by the surgical trauma team. Follow-up one year later showed no further syncopal episodes.

\section{Discussion}

Apical hypertrophic cardiomyopathy (AHC) is a rare variant of hypertrophic cardiomyopathy first described in Asian patients. ${ }^{1,3}$ It is more common in Asia, although it is also seen in the Western nations. ${ }^{2}$ It is predominantly a hereditary disease, although it can also be present in patients with no family history. ${ }^{4-6}$ In our case the patient was adopted, and no information related to his biological family was available. In the Japanese population AHC accounts for about $13 \%$ to $25 \%$ of the cases of hypertrophic cardiomyopathy, ${ }^{7}$ but it is less prevalent in the western population. ${ }^{8}$ Diagnostic modalities include ECG, ventriculography, nuclear myocardial perfusion studies, and MRI, which is emerging as the preferred modality. ${ }^{8-10}$

Apical hypertrophic cardiomyopathy can be asymptomatic or present with syncope, chest pain (symptoms similar to those of acute coronary syndromes have been described), palpitations, and dyspnea. ${ }^{11}$ In western patients there seems to be a varied presentation as far as clinical and ECG features are concerned, compared to the classic AHC as defined in the Asian population. ${ }^{11-13}$ Given its diverse presentation forms, plain clinical evaluation alone cannot be relied upon. In such situations, understanding the unique ECG features of AHC can be of assistance in the diagnostic process of this uncommon condition.

The presence of ECG findings indicative of left ventricular hypertrophy with giant T-wave inversions (especially in precordial leads) and loss of septal Q-wave should raise strong suspicions of AHC. These are considered pathognomnic for this disorder. ${ }^{1,14}$ The lack of septal Q-waves is thought to be due to intra-ventricular conduction defects, whereas the giant T-wave inversions are thought to result from the reversal of repolarization order within the distal left ventricular septum and apex. The reason for the loss of Q-waves is thought to be dysfunctional activation of the ventricle, but the exact mechanism is unknown. ${ }^{1}$ Although giant T-wave inversions in the setting of left ventricular hypertrophy are considered pathognomnic for $\mathrm{AHC}$, it is relevant to have a low threshold to rule out other more frequent causes of ST-T wave abnormalities that can present with syncopal episodes as well, such as coronary heart disease, neurological diseases (eg, subarachnoid hemorrhage), medication effect (eg, digoxin), and conditions with electrolyte derrangements (eg, 
hypomagnesaemia). Based on the numerous potential differential diagnoses to consider, comprehensive evaluation by cardiac imaging is necessary to confirm the diagnosis of $\mathrm{AHC}$. $^{8-10}$

Echocardiography has been universally accepted as the firstline imaging modality in investigation of patients with suspected AHC, due to its non-invasiveness, versatility, and well-established cost/benefit ratio. This continues to be true, despite its being an operator-dependent study, as well as the significant variation that might exist between the different exploratory windows. ${ }^{15}$ Use of contrast-enhanced echocardiography can be an option in an effort to better delineate the anatomy, minimizing the above-described limitations. ${ }^{16,17}$ A case in point is the better visualization of the left ventricular apical hypertrophy and aneurysm seen in our patient, compared to standard echocardiography, in which the apical aneurysm could not be well appreciated. Cardiac MRI is now emerging as "the gold standard" study in the diagnostic process of AHC given the limitations of echocardiography, the superiority in the assessment of the left ventricular anatomy, and its ability to detect the disease in early stages. ${ }^{8,9,18,19}$

Although the prevailing consensus is that $\mathrm{AHC}$ has a benign prognosis, ${ }^{11-14}$ there is emerging data showing increased risk for sudden cardiac arrest, fatal arrhythmias, heart failure, and ischemic events in patients with AHC. ${ }^{19-25}$ A recent study showed a substrate for monomorphic ventricular tachycardia to exist within the hypertrophied muscle component of AHC. ${ }^{24}$ Management of this condition includes watchful waiting, medical treatment, or invasive interventions such as myomectomy, arrhythmia ablation, and intracardiac cardioverter defibrillator (ICD) placement. ${ }^{24-26}$

Although there are no randomized controlled trials specifically in AHC populations, the management of each patient relies on the risk stratification as specified in the $2002 \mathrm{ACC} / \mathrm{ESC} /$ HRS practice guidelines for management of hypertrophic cardiomyopathy. ${ }^{27}$ Risk stratification in the general hypertrophic cardiomyopathy $(\mathrm{HCM})$ population is aimed at identifying individuals who are at high risk for sudden cardiac death (SCD) and would benefit from ICD placement. Factors associated with high risk of SCD among patients with HCM are: recurrent unexplained syncope, ventricular tachyarrhythmias, massive hypertrophy (wall thickness of 30 $\mathrm{mm}$ or more), previous cardiac arrest, abnormal blood pressure response on treadmill stress test, high-risk mutant gene, and family history of premature HCM-related sudden death. It is recommended that all patients with HCM undergo an annual risk assessment to evaluate for presence of any of these factors. ${ }^{27}$ Presence of each of these factors in a patient under the age of 60 years, denotes high risk for SCD and is, hence, an indication for ICD placement. This is consistent with the $2008 \mathrm{ACC} / \mathrm{ESC} / \mathrm{HRS}$ practice guidelines for devicebased therapy of cardiac rhythm abnormalities. ${ }^{27-31}$ Our patient was 47-years-old and had recurrent, unexplained syncope which placed him at high risk for SCD; for this reason ICD placement was indicated.

In addition to the features mentioned previously, another factor that has an effect on cardiovascular outcome is the presence of apical aneurysm, which our patient had. Patients with AHC and apical aneurysm have been observed to be at higher risk of SCD, embolic stroke, and progressive cardiac failure. Although not actually recognized as an independent risk factor for indication of ICD placement, its presence should be taken into consideration when a decision about ICD placement is being made. There are limited data on whether these patients might also benefit from chronic anticoagulation in order to reduce the risk of embolic phenomena. ${ }^{14,25,32}$

Beta-adrenergic blocking (BB) agents were among the first drugs used for the treatment of symptomatic HCM (heart failure symptoms, chest pain, and arrhythmias). The real benefit of $\mathrm{BB}$ remains debatable, due to lack of large randomized trials. It could even be questionable in the AHC subgroup, especially in absence of any other indication for its use, such as systemic hypertension, cardiac arrhythmias, or coronary heart disease. Until now, use of BB and nondihydropyridine calcium channel blockers has been, and remains, the initial drug treatment of choice for symptomatic patients, with the aim of alleviating symptoms. ${ }^{29}$ Potential mechanisms of these benefits include their capacity to reduce heart rate at rest and exercise, negative inotropy, and the reduction on left ventricular wall stress, which consequently reduces myocardial oxygen consumption. Patients' responses remain variable, and the role of $\mathrm{BB}$ in asymptomatic individuals is far from established and is empiric at best. In our case, the presence of angiographically significant coronary artery disease and the concern of possible underlying ventricular tachyarrhythmia justified the use of BB. In such patients there has been demonstrable clinical benefit. ${ }^{27,29}$

A small group of patients with HCM are considered to be candidates for surgical intervention (myectomy). This includes patients with marked septal hypertrophy and left ventricular outflow tract obstruction, presenting with severe exertional dyspnea, NYHA class III-IV heart failure, and refractoriness to maximum medical therapy. In these types of cases the main goal is to eliminate the portion of ventricular muscle (normally the interventricular septum) that is generating the obstruction. Although some reports are available, very little is known about the utility and benefit of myectomy in patients with the AHC variant. ${ }^{26,27,29}$ Other non-pharmacological therapies for the treatment of patients with HCM, like dual chamber pacing and ethanol septal ablation, were designed specifically (and only) to be used in the subset of patients where the site and extent of cardiac hypertrophy obstructs the left ventricular outflow. Based on the particular anatomical conditions necessary to implement these therapies, there is no room for their use in patients with the AHC variant where, by definition, no outflow tract obstruction is observed. 
In 1895, Willem Einthoven reported the first accurate recording of the ECG and introduced its utility as a clinical tool. Since then the ECG has become a fundamental element in the daily diagnostic process of cardiac diseases. This is a simple study, easy to do, very available, and fairly simple to interpret, with an excellent cost/benefit ratio; in other words, is a very low cost study, and the information that it provides is invaluable. In our case, the diagnosis was almost completely established with only the classical ECG findings that had been previously overlooked. We believe that the consequence of a missed diagnosis for this patient resulted in multiple traumatic events that could have been avoided if the appropriate interpretation of the ECG had been achieved in a timely fashion. This case demonstrates a very infrequent disease in our population, and at the same time underscores the vital emphasis that must be placed on training programs, primary care physicians, and other providers involved in the evaluation and treatment of patients with syncope, in developing appropriate and adequate ECG interpretation skills.

\section{Acknowledgments}

The authors wish to thank the Marshfield Clinic Research Foundation's Office of Scientific Writing and Publication for editorial assistance in the preparation of this manuscript.

\section{References}

1. Sakamoto T, Tei C, Murayama M, Ichiyasu H, Hada Y. Giant T wave inversion as a manifestation of asymmetrical apical hypertrophy (AAH) of the left ventricle. Echocardiographic and ultrasono-cardiotomographic study. Jpn Heart J 1976;17:611-629.

2. Kitaoka H, Doi Y, Casey SA, Hitomi N, Furuno T, Maron BJ. Comparison of prevalence of apical hypertrophic cardiomyopathy in Japan and the United States. Am J Cardiol 2003;92:1183-1186.

3. Yamaguchi H, Ishimura T, Nishiyama S, Nagasaki F, Nakanishi S, Takatsu F, Nishijo T, Umeda T, Machii K. Hypertrophic nonobstructive cardiomyopathy with giant negative $\mathrm{T}$ waves (apical hypertrophy): ventriculographic and echocardiographic features in 30 patients. Am J Cardiol 1979;44:401-412.

4. Arad M, Penas-Lado M, Monserrat L, Maron BJ, Sherrid M, Ho CY, Barr S, Karim A, Olson TM, Kamisago M, Seidman JG, Seidman CE. Gene mutations in apical hypertrophic cardiomyopathy. Circulation 2005;112:2805-2811.

5. Monserrat L, Hermida-Prieto M, Fernandez X, Rodríguez I, Dumont C, Cazón L, Cuesta MG, Gonzalez-Juanatey C, Peteiro J, Alvarez N, Penas-Lado M, Castro-Beiras A. Mutation in the alpha-cardiac actin gene associated with apical hypertrophic cardiomyopathy, left ventricular noncompaction, and septal defects. Eur Heart J 2007; 28:1953-1961.

6. Abdy NA, Valdes SO, Sorrell VL, Klewer SE, Barber BJ. Apical hypertrophic cardiomyopathy in an adolescent. Congenit Heart Dis 2010;5:182-187.

7. Yamaguchi H, Nishiyama S, Nakanishi S, Nishimura S. Electrocardiographic, echocardiographic and ventriculographic characterization of hypertrophic nonobstructive cardiomyopathy. Eur Heart J 1983;4 Suppl F: 105-119
8. Moon JC, Fisher NG, McKenna WJ, Pennell DJ. Detection of apical hypertrophic cardiomyopathy by cardiovascular magnetic resonance in patients with non-diagnostic echocardiography. Heart 2004;90:645-649.

9. Fattori R, Biagini E, Lorenzini M, Buttazzi K, Lovato L, Rapezzi C. Significance of magnetic resonance imaging in apical hypertrophic cardiomyopathy. Am J Cardiol 2010;105:1592-1596.

10. Cianciulli TF, Saccheri MC, Masoli OH, Redruello MF, Lax JA, Morita LA, Gagliardi JA, Dorelle AN, Prezioso HA, Vidal LA. Myocardial perfusion SPECT in the diagnosis of apical hypertrophic cardiomyopathy. J Nucl Cardiol 2009;16:391-395.

11. Chen CH, Nobuyoshi M, Kawai C. ECG pattern of left ventricular hypertrophy in nonobstructive hypertrophic cardiomyopathy: the significance of the mid-precordial changes. Am Heart J 1979;97:687-695.

12. Lee CH, Liu PY, Lin LJ, Chen JH, Tsai LM. Clinical features and outcome of patients with apical hypertrophic cardiomyopathy in Taiwan. Cardiology 2006;106:29-35.

13. Chung T, Yiannikas J, Freedman SB, Kritharides L. Unusual features of apical hypertrophic cardiomyopathy. Am J Cardiol 2010;105:879-883.

14. Eriksson MJ, Sonnenberg B, Woo A, Rakowski P, Parker TG, Wigle ED, Rakowski H. Long-term outcome in patients with apical hypertrophic cardiomyopathy. J Am Coll Cardiol 2002;39:638-645.

15. Thanigaraj S, Pérez JE. Apical hypertrophic cardiomyopathy: echocardiographic diagnosis with the use of intravenous contrast image enhancement. J Am Soc Echocardiogr 2000;13:146-149.

16. Moukarbel GV, Alam SE, Abchee AB. Contrast-enhanced echocardiography for the diagnosis of apical hypertrophic cardiomyopathy. Echocardiography 2005;22:831-833.

17. Pons-Lladó G, Carreras F, Borrás X, Palmer J, Llauger J, Bayés de Luna A. Comparison of morphologic assessment of hypertrophic cardiomyopathy by magnetic resonance versus echocardiographic imaging. Am J Cardiol 1997; 79:1651-1656.

18. Suzuki J, Shimamoto R, Nishikawa J, Yamazaki T, Tsuji T, Nakamura F, Shin WS, Nakajima T, Toyo-Oka T, Ohotomo $\mathrm{K}$. Morphological onset and early diagnosis in apical hypertrophic cardiomyopathy: a long term analysis with nuclear magnetic resonance imaging. J Am Coll Cardiol 1999;33:146-151.

19. Sakamoto T. Apical hypertrophic cardiomyopathy (apical hypertrophy): an overview. J Cardiol 2001;37 Suppl 1:161-178.

20. Ridjab D, Koch M, Zabel M, Schultheiss HP, Morguet AJ. Cardiac arrest and ventricular tachycardia in Japanese-type apical hypertrophic cardiomyopathy. Cardiology 2007;107:81-86.

21. Carter D, Pokroy R, Barenboim E, Azaria B, Goldstein L. Apical hypertrophic cardiomyopathy and arrhythmia in military pilots. Aviat Space Environ Med 2006;77:459-461.

22. Okishige K, Sasano T, Yano K, Azegami K, Suzuki K, Itoh K. Serious arrhythmias in patients with apical hypertrophic cardiomyopathy. Intern Med 2001;40:396-402.

23. Suganuma Y, Shinmura K, Hasegawa H, Tani M, Nakamura Y. Clinical characteristics and cardiac events in elderly patients with apical hypertrophic cardiomyopathy. Nippon Ronen Igakkai Zasshi. [Article in Japanese] 1997;34:474-481.

24. Inada K, Seiler J, Roberts-Thomson KC, Steven D, Rosman J, John RM, Sobieszczyk P, Stevenson WG, Tedrow UB. Substrate characterization and catheter ablation for monomorphic ventricular tachycardia in patients with apical hypertrophic cardiomyopathy. J Cardiovasc Electrophysiol 2011;22:41-48. 
25. Sanghvi NK, Tracy CM. Sustained ventricular tachycardia in apical hypertrophic cardiomyopathy, midcavitary obstruction, and apical aneurysm. Pacing Clin Electrophysiol 2007;30:799-803.

26. Schaff HV, Brown ML, Dearani JA, Abel MD, Ommen SR, Sorajja P, Tajik AJ, Nishimura RA. Apical myectomy: a new surgical technique for management of severely symptomatic patients with apical hypertrophic cardiomyopathy. J Thorac Cardiovasc Surg 2010;139:634-640.

27. Maron BJ, McKenna WJ, Danielson GK, Kappenberger LJ, Kuhn HJ, Seidman CE, Shah PM, Spencer WH 3rd, Spirito P, Ten Cate FJ, Wigle ED; American College of Cardiology Foundation Task Force on Clinical Expert Consensus Documents; European Society of Cardiology Committee for Practice Guidelines. American College of Cardiology/ European Society of Cardiology Clinical Expert Consensus Document on Hypertrophic Cardiomyopathy. A report of the American College of Cardiology Foundation Task Force on Clinical Expert Consensus Documents and the European Society of Cardiology Committee for Practice Guidelines. Eur Heart J 2003;24:1965-1969.

28. Epstein AE, DiMarco JP, Ellenbogen KA, Estes NA 3rd, Freedman RA, Gettes LS, Gillinov AM, Gregoratos G, Hammill SC, Hayes DL, Hlatky MA, Newby LK, Page RL, Schoenfeld MH, Silka MJ, Stevenson LW, Sweeney MO, Smith SC Jr, Jacobs AK, Adams CD, Anderson JL, Buller CE, Creager MA, Ettinger SM, Faxon DP, Halperin JL, Hiratzka LF, Hunt SA, Krumholz HM, Kushner FG, Lytle BW, Nishimura RA, Ornato JP, Page RL, Riegel B, Tarkington LG, Yancy CW; American College of Cardiology/ American Heart Association Task Force on Practice Guidelines (Writing Committee to Revise the ACC/AHA/ NASPE 2002 Guideline Update for Implantation of Cardiac Pacemakers and Antiarrhythmia Devices); American Association for Thoracic Surgery; Society of Thoracic Surgeons. ACC/AHA/HRS 2008 Guidelines for DeviceBased Therapy of Cardiac Rhythm Abnormalities: a report of the American College of Cardiology/American Heart Association Task Force on Practice Guidelines (Writing Committee to Revise the ACC/AHA/NASPE 2002 Guideline Update for Implantation of Cardiac Pacemakers and Antiarrhythmia Devices) developed in collaboration with the American Association for Thoracic Surgery and Society of Thoracic Surgeons. Circulation 2008;117:e350-408.

29. Spirito P, Seidman CE, McKenna WJ, Maron BJ. The management of hypertrophic cardiomyopathy. N Engl J Med 1997;336:775-785.

30. Maron BJ, Spirito P, Shen WK, Haas TS, Formisano F, Link MS, Epstein AE, Almquist AK, Daubert JP, Lawrenz T, Boriani G, Estes NA 3rd, Favale S, Piccininno M, Winters SL, Santini M, Betocchi S, Arribas F, Sherrid MV, Buja G, Semsarian C, Bruzzi P. Implantable cardioverter-defibrillators and prevention of sudden cardiac death in hypertrophic cardiomyopathy. JAMA 2007;298(4):405-412.

31. Maron BJ, Spirito P. Implantable defibrillators and prevention of sudden death in hypertrophic cardiomyopathy. $\mathrm{J}$ Cardiovasc Electrophysiol 2008;19:1118-1126.

32. Maron MS, Finley JJ, Bos JM, Hauser TH, Manning WJ, Haas TS, Lesser JR, Udelson JE, Ackerman MJ, Maron BJ. Prevalence, clinical significance, and natural history of left ventricular apical aneurysms in hypertrophic cardiomyopathy. Circulation 2008;118:1541-1549.

\section{Author Affiliations}

Yusuf Kasirye, MD*,1; Janaki R. Manne, MD'; Narendranath Epperla, MD*; Sowjanya Bapani, MD*; Romel GarciaMontilla, $M D^{\prime}$

*Department of Internal Medicine, Marshfield Clinic, Marshfield WI, 54449

Department of Hospital Medicine, Marshfield Clinic, Marshfield, WI 54449

'Current affiliation: Department of Internal Medicine, Marshfield Clinic, Park Falls, WI 54552 